\section{A New Surgical Barbed Staple for Minimally Invasive Surgery ${ }^{1}$}

\author{
Farhad Javid $^{2}$ \\ Centre for Intelligent Machines (CIM) and \\ Department of Mechanical Engineering, \\ McGill University, \\ Montreal, QC H3A 0C3, Canada \\ e-mail: farhad@cim.mcgill.ca

\section{Damiano Pasini} \\ Department of Mechanical Engineering, \\ McGill University, \\ Macdonald Engineering Building, \\ 817 Sherbrooke St. West, \\ Montreal, QC H3A 2K6, Canada

\section{Jorge Angeles} \\ Department of Mechanical Engineering, \\ McGill University, \\ McConnell Engineering Building 452, \\ 817 Sherbrooke St. West, \\ Montreal, QC H3A 2K6, Canada
}

\section{Background}

Standard suturing is an inevitable but time-consuming procedure in all surgical operations. In minimally invasive (MI) surgery, since the complex knot-tying maneuvers are extremely tedious, suturing is even more challenging. Knotless fixation implants are, therefore, developed to eliminate the conventional suturing procedure in surgical operations. Barbed sutures [1], surgical staples, and self-closing clips [2] are widely used in open surgical operations. Moreover, special surgical systems such as the Endo Stitch device, the SILS Stitch articulating device, and the Multifire Endo GIA stapler are designed to replace sutures in a variety of MI operations. These devices, however, are not suitable for precise MI surgery in small operation sites since they require considerably large insertion holes and extensive operating cavities. A novel surgical barbed staple and its insertion system, to be used in precise MI operations, are introduced in this work.

\section{Conceptual Design}

The idea of a barbed staple is derived from the well-known barbed suture developed for cosmetic surgery [3]. Directional projections or barbs are cut along a monofilament suture to anchor the surrounding tissue after insertion and fix the suture inside the tissue. The first surgical barbed staple was proposed in a patent [4] for cosmetic surgery. Two new designs for the barbed staple are introduced in this work. As shown in Fig. 1, the first design consists of a rigid core and a flexible sheath. The rigid core gives the staple the strength required to pierce the tissue during insertion. The sheath is a flexible cover surrounding the staple core. The barbs are cut along the sheath of the staple. Since the strength of the flexible sheath is close to that of the tissue, the barbs cannot easily cut the tissue and, therefore, the anchoring force of the staple is increased. Based on the application, the staple core and

\footnotetext{
${ }^{1}$ Accepted and presented at the Design of Medical Devices Conference (DMD2014), Minneapolis, MN, April 7-10, 2014.

DOI: $10.1115 / 1.4027118$

${ }^{2}$ Corresponding author

Manuscript received February 21, 2014; final manuscript received March 3, 2014 published online July 21, 2014. Editor: Arthur G. Erdman.
}

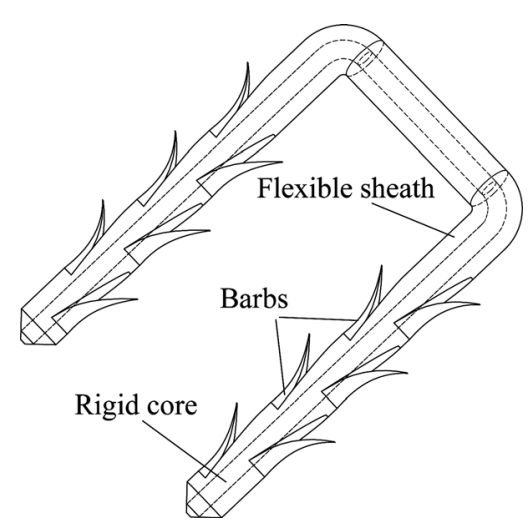

Fig. 1 The structure of the rigid barbed staple

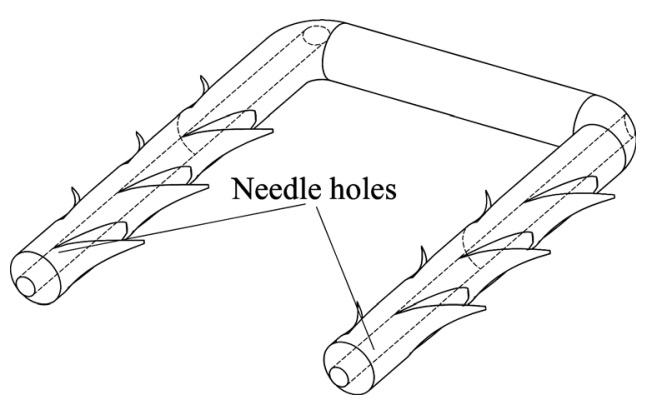

Fig. 2 The flexible barbed staple

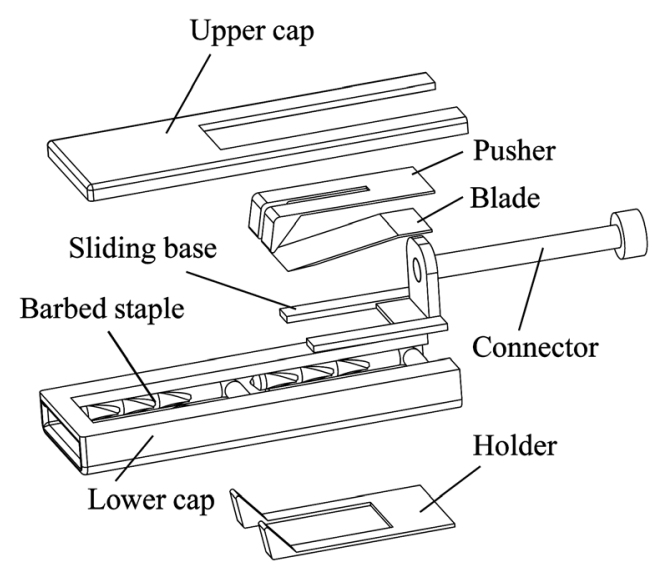

Fig. 3 Exploded views of the stapling mechanism

sheath can be fabricated of absorbable or nonabsorbable materials.

In the second design, the rigid core is removed and one bore is created in the middle of each staple leg, as shown in Fig. 2. Two needles are inserted in the staple bores during the insertion process. The needles give the flexible suture enough rigidity to penetrate the tissue. Since the barbs anchor the surrounding tissue, the staple remains in the tissue after retracting the needles.

\section{Insertion Method}

The flexible staple is readily inserted using a pair of insertion needles passed through the staple holes. A new microstapling mechanism is, moreover, designed for the insertion of the rigid staple. The mechanism consists of an upper and a lower cap, a holder, a pusher, a cutting blade, and a sliding base. The exploded view of the stapling mechanism is shown in Fig. 3. 


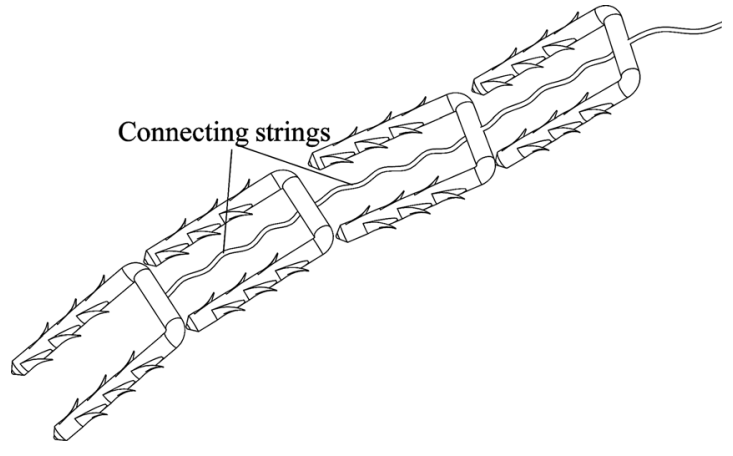

Fig. 4 Staples interconnected with monofilament absorbable strings

The staples are first interconnected using an absorbable monofilament string, as shown in Fig. 4. The interconnected staples are aligned inside the microstapler conveyor. The latter is a tube with rectangular cross section, connected to the stapling mechanism, and acts as the staple reservoir during the operation. The staples are easily moved in the conveyor and fed into the stapling mechanism mounted on the tip of the conveyor. The connecting strings cause the staples to move forward while the first staple is inserting. The second staple is, therefore, placed on the tip of the mechanism after the insertion of the first one.

Before insertion, the tip of the mechanism is placed perpendicular to the tissue and slightly presses against the tissue to make the required contact force for insertion. At each shot, the pusher inserts the first barbed staple into the tissue and the blade cuts the connecting string to detach the inserted staple from the remaining part, while the next staple is placed on the tip of the mechanism, ready for insertion. The spring pusher is initially placed behind the first staple and pushes it toward the tissue at the surgeon's command. The sharp tips of the staple legs cut through the tissue while the barbs gradually anchor the surrounding tissue and prevent it from retracting. Since the staples are interconnected, they all move when the first one is pushed. The second staple is therefore placed on the tip of the catheter while the first one is inserted. At the end of the insertion step, the blade hits the anvil section in the upper cap and is bent downward, cutting the connecting string between the first two staples and releasing the first one. When the surgeon releases the trigger, the mechanism returns to its original position. During this step, the spring pusher is bent to pass the staple placed on the tip of the mechanism.

\section{Conclusions}

Two novel barbed staples and a microstapler mechanism are designed, as reported here, to replace the tedious suturing procedure in MI operations. Since the barbs anchor the surrounding tissue after insertion, it is not required to bend the staple legs to fix it inside the tissue. The microstapler mechanism is, therefore, smaller and the insertion procedure is simpler.

\section{References}

[1] Einarsson, J. I., Cohen, S. L., Gobern, J. M., Sandberg, E. M., Hill-Lydecker, C. I., Wang, K., and Brown, D. N., 2013, "Barbed Versus Standard Suture: A Randomized Trial for Laparoscopic Vaginal Cuff Closure," J. Minimally Invasive Gynecol., 20(4), pp. 492-498.

[2] Baron, T. H., Song, L. M. W. K., Ross, A., Tokar, J. L., Irani, S., and Kozarek, R. A., 2012, "Use of an Over-the-Scope Clipping Device: Multicenter Retrospective Results of the First U.S. Experience (With Videos)," Gastrointest. Endoscopy, 76(1), pp. 202-208.

[3] Jandali, S., Nelson, J. A., Bergey, M. R., Sonnad, S. S., and Serletti, J. M., 2011, "Evaluating the Use of a Barbed Suture for Skin Closure During Autologous Breast Reconstruction,” J. Reconstr. Microsurg., 27(5), pp. 277-286.

[4] Romans, M. L., 2010, “Absorbable Surgical Staple," US Patent 2010-0256675 A1, CA. 